University of Nebraska - Lincoln

DigitalCommons@University of Nebraska - Lincoln

Ralph Skomski Publications

Research Papers in Physics and Astronomy

July 2007

\title{
Finite-temperature depolarization in half metals
}

\author{
Ralph Skomski \\ University of Nebraska-Lincoln, rskomski2@unl.edu
}

Follow this and additional works at: https://digitalcommons.unl.edu/physicsskomski

Part of the Physics Commons

Skomski, Ralph, "Finite-temperature depolarization in half metals" (2007). Ralph Skomski Publications. 45. https://digitalcommons.unl.edu/physicsskomski/45

This Article is brought to you for free and open access by the Research Papers in Physics and Astronomy at DigitalCommons@University of Nebraska - Lincoln. It has been accepted for inclusion in Ralph Skomski Publications by an authorized administrator of DigitalCommons@University of Nebraska - Lincoln. 
Published in Journal of Physics: Condensed Matter 19 (2007) 315202 (14 pp); doi:10.1088/09538984/19/31/315202 Online at http://stacks.iop.org/JPhysCM/19/315202 Copyright (C) 2007 IOP Publishing Ltd. Used by permission.

Submitted November 14, 2006; revised April 23, 2007; published July 3, 2007.

\title{
Finite-temperature depolarization in half metals
}

\author{
R. Skomski \\ Department of Physics and Astronomy and Nebraska Center for Materials and \\ Nanoscience, University of Nebraska-Lincoln, Lincoln, NE 68588, USA
}

\begin{abstract}
The temperature dependence of the spin polarization of type-I half-metallic ferromagnets is investigated and compared with that of other magnetic materials, such as semimetals, strong and weak ferromagnets, and exchange-enhanced Pauli paramagnets. Stable atomic moments, as realized by strong intra-atomic exchange, exhibit a nonzero spin-down density of states (DOS) at finite temperatures. This thermal spin mixing means that the conductivity of the "insulating" spin channel is always nonzero and that half-metallic ferromagnetism is an idealized limit. At zero temperature, similar effects are caused by intersublattice interactions, spin-orbit coupling and crystal imperfections. With increasing interatomic hopping, the moment becomes unstable, and Stoner-type thermal excitations yield an additional reduction of the spin polarization. In the Stoner limit, the hybridization gap closes far below the Curie temperature, and the corresponding transition temperature $T^{*}$ increases with increasing hybridization gap and decreasing band width. Correlations are analyzed by a version of the Kondo model and by an unrestricted Hartree-Fock approximation, and it is argued that correlations are less important than the leading one-electron contributions.
\end{abstract}

\section{Introduction}

One approach in spin electronics is to use half-metallic ferromagnets or half metals [13]. Ideal band gap half metals have nonzero $\uparrow$ and zero $\downarrow$ densities of states (DOSs) at the Fermi level, or vice versa, so there is only one spin channel for conduction. This translates into a spin-dependent conductivity and can be exploited in various applications. A key aspect of half-metallic ferromagnetism is finite-temperature behavior of the spin polarization, especially at the Fermi level. Both experiment and theory indicate that finitetemperature excitations are very effective in destroying the spin polarization. A striking experimental feature is the onset of "normal" ferromagnetism above $80-100 \mathrm{~K}$ in the semiHeusler alloy NiMnSb [4] and in $\mathrm{CrO}_{2}$ above 50-100 K [5]. Furthermore, real-structure features such as defects, surfaces, and interfaces may drastically reduce the spin polarization [3]. Electron states in half metals may be itinerant or localized, but even in the itinerant case, the finite-temperature behavior exhibits localized features [6], very similar to the situation encountered in ordinary itinerant ferromagnets $[7,8]$. 


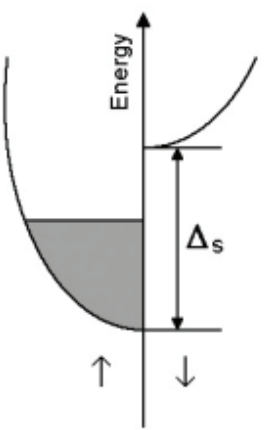

(a)

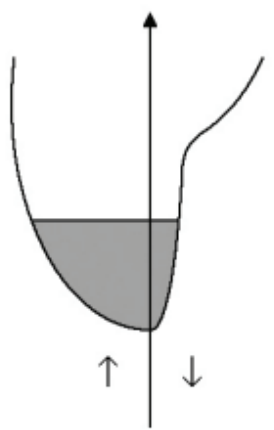

(b)

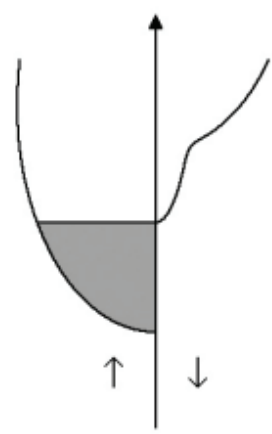

(c)

Figure 1. Schematic density of states in a type- $\mathrm{I}_{B}$ half metal: (a) one-electron ground state, (b) effect of spin mixing at $T>0$ and (c) inclusion of non-quasiparticle states at $T=0$.

A number of experimental and theoretical aspects of half metallics and some specific materials have been reviewed recently [2, 3, 9-12]. This paper focuses on the finite-temperature behavior of typical hybridization-gap half metals and includes both band-structure and correlation effects. One question is how the density of states (DOS) is modified. Figure 1 illustrates this point by comparing typical densities of states. Figure 1(a) shows the zero-temperature $\uparrow$ and $\downarrow$ densities of states $D(E)$ for a typical half metal. The DOS is characterized by a spin splitting $\Delta \mathrm{s}$, and we see that $D\left(E_{\mathrm{F}}\right)=0$ for $\downarrow$ electrons. Figure 1 (b) illustrates the modification of the DOS due to thermally activated spin mixing [13, 14]. We see that this mechanism yields a nonzero $D\left(E_{\mathrm{F}}\right)$ for $\downarrow$ electrons. Finally, figure $1(\mathrm{c})$ shows how the zero-temperature DOS is modified by a different mechanism, namely electron-magnon interactions leading to nonquasiparticle states $[12,15]$. Here the $\downarrow$ DOS remains nonzero, but the onset of a nonzero $\downarrow$ DOS just above the Fermi level indicates that these excitations may become important at finite temperatures.

As analyzed by Skomski and Dowben [13], the reduction of the spin polarization due to spin mixing reflects the temperature dependence of the magnetization. For one-component magnets, a simple formula was obtained, and it was mentioned that crystal-specific features modify the picture $[3,13]$. The same authors concluded that noncollinear sublattice effects play an important role in the realization of the spin polarization $[3,16]$. More recently, a first-principles study by the Blügel group [17] has emphasized the role of hybridization effects, in addition to confirming the importance of local and global noncollinearities.

The main focus of this review is on how intra-atomic exchange and interatomic hopping modify the density of states and affect the finite-temperature behavior of half-metallic ferromagnets. A more practical question is: what structural and electronic features determine the reduction of the spin polarization at finite temperatures? Throughout the paper, we will compare half-metallic ferromagnetism with other types of magnetically ordered solids, such as itinerant ferromagnets and antiferromagnets, semimetals, Kondo magnets, and very weak itinerant ferromagnets.

\section{Half metals and related magnetic materials}

\subsection{Classification of half metals}

Half metals have been classified by Coey and Venkatesan [2]. The are five main classes (I-V), each divided into subclasses A and B depending on whether the conductivity is re- 


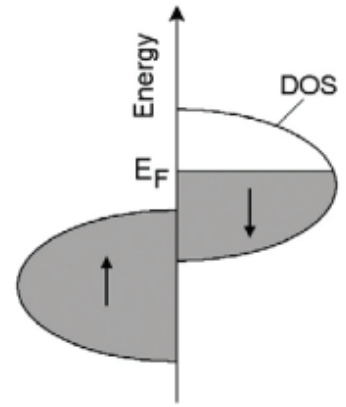

(a)

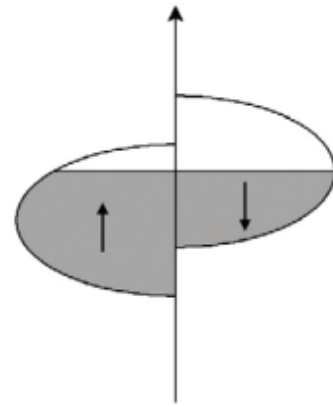

(b)

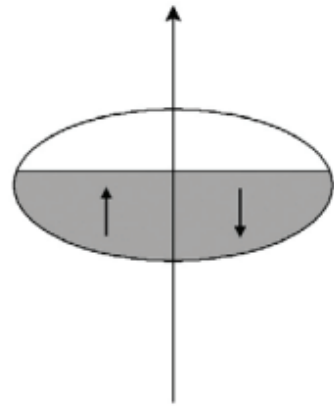

(c)

Figure 2. Simplified Stoner-type picture of itinerant ferromagnetism: (a) strong ferromagnet, (b) weak ferromagnet, and (c) Pauli paramagnet. Transitions from (a) to (b) and (c) may be realized, for example, by lattice compression, but thermally activated transitions to (c) are uncommon in simple ferromagnets.

alized by $\uparrow$ electrons (A) or by $\downarrow$ electrons (B). Type-I half metals (delocalized hybridization-gap materials) are similar to strong ferromagnets but are characterized by the absence of $4 \mathrm{~s}$ electrons at the Fermi level. Typical examples are $\mathrm{CrO}_{2}$ (IA), NiMnSb (IA), and $\mathrm{Mn}$ ${ }_{2} \mathrm{VAl}$ (IB), and a schematic IA DOS is shown in figure 1(a). Type-II half metals (localized hybridization-gap materials) such as $\mathrm{Fe}_{3} \mathrm{O}_{4}$ (IIA) are nonmetallic due to narrow $3 \mathrm{~d}$ bands but otherwise similar to type-I half metals. Their conductivity reflects the thermally activated hopping of $\uparrow$ (IIA) and $\downarrow$ (IIB) electrons.

In type-III half metals, or transport half metals, $D\left(E_{\mathrm{F}}\right)>0$ for both $\uparrow$ and $\downarrow$ electrons, but for one spin direction, the electrons are localized. An example is the manganite $\mathrm{La}_{0.7} \mathrm{Sr}_{0.3} \mathrm{MnO}_{3}$, which contains mobile Mn electrons of $\mathrm{e}_{\mathrm{g}}$ character. Finally, most semimetals and semiconductors are nonmagnetic, but some carry a ferromagnetic moment and can be classified as type-IV and type-V half metallics, respectively. Semimetals, such as $\mathrm{Bi}$, are defined by small carrier densities due to marginal overlap between valence and conduction bands. Unlike semiconductors, they have no band gaps, but the low carrier densities characteristic of both types of material lead to relatively large ranges of magnetic interactions $[18,19]$.

\subsection{Relation to strong and weak ferromagnetism}

A key aspect of the finite-temperature depolarization is the nature of the excitations that reduce the spin polarization. Half-metallic ferromagnetism is closely related to strong ferromagnetism, except that ordinary strong ferromagnets, such as Co and $\mathrm{Ni}$, have $\uparrow$ and $\downarrow 4 \mathrm{~s}$ electrons at the Fermi level. Figure 2 compares schematic densities of states (DOSs) for strong ferromagnets, weak ferromagnets, and Pauli paramagnets. The figure epitomizes the interpretation of half-metallic ferromagnetism as a delocalized or itinerant phenomenon.

Simplifying somewhat, itinerant ferromagnetism is caused by the Coulomb interaction $V_{12}\left(\mathbf{r}, \mathbf{r}^{\prime}\right)=\mathrm{n}_{\uparrow}(\mathbf{r}) \mathrm{n}_{\downarrow}\left(\mathbf{r}^{\prime}\right) / 4 \pi \varepsilon_{o}\left|\mathbf{r}-\mathbf{r}^{\prime}\right|$ between the electrons, where $\mathrm{n}_{\uparrow}$ and $\mathrm{n}_{\downarrow}$ are the particle-number operators for the two spin orientations. In this expression, it is not necessary to consider pairs of $\uparrow \uparrow$ and $\downarrow \downarrow$ electrons, because the Pauli principle forbids the occupancy of any state by electrons of parallel spin. Introducing the total particle number $n=n_{\uparrow}+n_{\downarrow}$ and the spin polarization $s=n_{\uparrow}-n_{\downarrow}$, one obtains that $n_{\uparrow} n_{\downarrow}=\left(n^{2}-s^{2}\right) / 4$. This shows that the Stoner exchange favors ferromagnetism, $\mathbf{s}>0$. Depending on the context, this mechanism is known as intra-atomic exchange, Stoner exchange, or Hund's rule exchange. 
In the simplest case, itinerant magnetism is described by the Stoner theory. It uses the approximate relation

$$
\mathrm{n}_{\uparrow} \mathrm{n}_{\downarrow}=\mathrm{n}_{\uparrow}\left\langle n_{\downarrow}\right\rangle+\mathrm{n}_{\downarrow}\left\langle n_{\uparrow}\right\rangle
$$

to map the complicated many-electron interactions $V_{12}\left(\mathbf{r}, \mathbf{r}^{\prime}\right)$ on a effective or mean field $V_{\text {eff }}(\mathbf{r}) \sim I\langle s\rangle$. Here the Stoner parameter $I \sim \int V_{12}\left(\mathbf{r}, \mathbf{r}^{\prime}\right) \mathrm{d} V \mathrm{~d} V^{\prime}$ describes the strength of intra-atomic Coulomb interaction. Essentially, the Stoner theory is a one-electron theory on a Hartree-Fock level, described by a single big Slater determinant. It ignores correlation effects which go - by definition - beyond the use of a single determinant. The Stoner interaction $I \approx 1 \mathrm{eV}$ competes against one-electron hybridization energies (hopping energies), which are described by the d-band width $W$ or, more precisely, by the density of states $D \sim$ $1 / W$. Pronounced hopping yields low-lying bonding states populated by $\uparrow \downarrow$ electron pairs, but in sufficiently narrow bands, the Stoner interaction dominates and the material becomes ferromagnetic. The corresponding condition $D\left(E_{\mathrm{F}}\right) I>1$ is known as the Stoner criterion, and, in the ferromagnetic phase, the spin splitting $D_{s}$ is proportional to $I$.

Figure 2 shows the vanishing of the magnetic moment in the itinerant limit. In some materials, transitions between figures $2(a)$, (b) and (c) can be tuned by varying the lattice parameter $[8,20]$, but thermally activated Stoner transitions are a different story. Thermal excitations may destroy the magnetic moment by smearing the Fermi level, but typical exchange splittings are of the order of $1 \mathrm{eV}$, corresponding to temperature differences of order $10,000 \mathrm{~K}$. In fact, the mechanism of figure 2 greatly overestimates the Curie temperature $T_{\mathrm{c}}$ of strong ferromagnets and makes the physically unreasonable prediction that the atomic moments vanish at $T_{\mathrm{c}}{ }^{1}$ The failure of the Stoner theory is due to spin fluctuations [7, 21-25], that is, $\mathbf{M}=0$ at $T_{\mathrm{c}}$ reflects the thermal randomization of atomic spin directions in combination with well-conserved atomic moments.

Weak ferromagnets are subclassified into nearly strong ferromagnets, such as Fe, and very weak itinerant (VWI) ferromagnets, such as $\mathrm{ZrZn}_{2}$ [25]. Nearly strong ferromagnets exhibit strong spin fluctuations and behave similarly to strong ferromagnets. In VWI ferromagnets, the Stoner criterion is barely satisfied, the ferromagnetism is unstable, and the magnetic moments are drastically reduced at the Curie temperature. In this rather exotic regime, thermal excitations yield a transition from weak ferromagnetism, figure 2(b), to metallic paramagnetism, figure 2(c). A similar subclassification exists for paramagnets, distinguishing ordinary Pauli paramagnets from exchange-enhanced Pauli paramagnets such as $\mathrm{Pd}$ and Pt. Exchange-enhanced paramagnets are close to satisfying the Stoner criterion and are easily spin polarized by magnetic neighbors. They exhibit nearly ferromagnetic regions (paramagnons) reminiscent of VWI spin fluctuations.

The localized (or Heisenberg) and delocalized (or Stoner) regimes in strong and VWI ferromagnets, respectively, are idealized limits. The real situation is intermediate, but the question arises whether half metals are closer to strong ferromagnetism or to VWI ferromagnetism. For half metals, the situation was analyzed by Otto et al. [6], who advocated the local-band picture, that is, strong ferromagnetism (section 3). However, this view has not remained unchallenged and seems to be only partially adequate (section 5).

\section{Spin mixing at finite temperatures}

The randomization of the spin directions in the localized picture involves Coulomb interactions on a local scale and is, in a strict sense, a many-body effect. However, essential features of the mechanism are reproduced on a one-electron level, by treating $\mathrm{n} \uparrow$ and $\mathrm{n} \downarrow$ as local operators with quantization axes parallel to the atomic magnetic moments $[7,20]$. This

\footnotetext{
${ }^{1}$ By definition, $T_{\mathrm{c}}$ implies the vanishing of the volume-averaged moment $\mathbf{M} \sim \mathbf{m}$, but this is usually realized as an average over all moment directions $\mathbf{m} / m$ whereas $m=|\mathbf{m}|$ remains finite.
} 


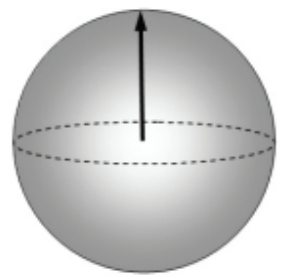

(a) $\mid \psi>=(1,0)$

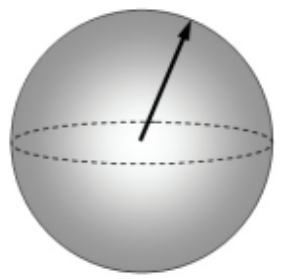

(b) $|\psi\rangle=\left(\psi_{\uparrow}, \psi_{\downarrow}\right)$

Figure 3. Spin directions and wavefunctions: (a) $T=0$ and (b) $T>0$. A classical vector pointing in a direction above the equator plane $(x-y$ plane) has no projection in the $-z$ direction, but in quantum mechanics there is a nonzero probability $\psi_{\downarrow}^{*} \psi_{\downarrow}$ of realizing the $\downarrow$ state.

does not mean that correlation effects are unimportant in half metals. One example is the Kondo effect, which is closely related to the double exchange in transport half metals such as $\mathrm{La}_{0.7} \mathrm{Sr}_{0.3} \mathrm{MnO}_{3}$ (section 5.3). The limit of strong intra-atomic exchange corresponds to the picture of figure 1(b) [13]. Interatomic hopping leads to conduction in the $\uparrow$ channel of a type- $\mathrm{I}_{\mathrm{B}}$ half metal if there are $\downarrow$ states at the Fermi level. These states are automatically created by spin disorder, because any misaligned spin has a nonzero projection in the $\uparrow$ direction (spin mixing). Figure 3 illustrates this quantum effect for isolated atoms.

A semiquantitative treatment of the spin mixing is achieved by specifying hopping, orbital and intra-atomic energies. A simple tight-binding model Hamiltonian is

$$
\mathcal{H}_{\mu \nu i k \sigma \sigma^{\prime}}=t_{\mu \nu i k} \delta_{\alpha \beta}-V_{\mu} \delta_{\mu \nu} \delta_{i k} \delta_{\alpha \beta}-I \delta_{\mu \nu} \delta_{i k} \mathbf{e}_{i} \cdot \sigma_{\alpha \beta} .
$$

Here $t_{\mu v i k}=\left\langle\varphi_{\mu}\left(\mathbf{r}-\mathbf{R}_{i}\right)|t| \varphi_{v}\left(\mathbf{r}-\mathbf{R}_{k}\right)\right\rangle$ is the hopping integral between a $\mu$-type orbital at $\mathbf{R}_{i}$ and a $v$-type orbital at $\mathbf{R}_{k}, V_{\mu}$ is the crystal-field energy for the $\mu$-type orbital, and $\mathbf{e}_{i}$ is the local magnetization direction. In the absence of spin disorder, the spin structure of a ferromagnet is described by the Pauli matrix $\sigma_{z}$ whose $\uparrow$ and $\downarrow$ eigenfunctions are $(1,0)^{\mathrm{T}}$ and $(0,1)^{\mathrm{T}}$, respectively, and yields an exchange-energy difference of $2 I$ between spin-up and spin-down electrons. The starting point for describing finite-temperature spin disorder is the standard spin- $1 / 2$ rotation matrix

$$
\mathrm{U}(\phi, \theta)=\left(\begin{array}{cc}
\cos \left(\frac{\theta}{2}\right) \mathrm{e}^{\mathrm{i} \phi / 2} & \sin \left(\frac{\theta}{2}\right) \mathrm{e}^{-\mathrm{i} \phi / 2} \\
-\sin \left(\frac{\theta}{2}\right) \mathrm{e}^{\mathrm{i} \phi / 2} & \cos \left(\frac{\theta}{2}\right) \mathrm{e}^{-\mathrm{i} \phi / 2}
\end{array}\right)
$$

which rotates a $\uparrow$ spin by the angles $\theta$ and $\varphi$. The $\mathbf{e}_{i} \cdot \sigma_{\alpha \beta}$ exchange term in (1) is diagonalized by $\mathrm{U}^{-1}=\mathrm{U}^{+}\left(\varphi_{i}, \theta_{i}\right)$. Applying the transformation $\bigcup_{t}=\mathrm{U}^{+}\left(\varphi_{1}, \theta_{1}\right) \cdots \mathrm{U}^{+}\left(\varphi_{i}, \theta i\right) \mathrm{U}^{+}\left(\varphi_{i}\right.$ $\left.+1, \theta_{i}+1\right) \cdots \mathrm{U}^{+}\left(\varphi_{N^{\prime}}, \theta_{N}\right)$ to equation (2) yields

$$
\mathcal{H}_{\mu \nu i k \alpha \beta}^{t}=t_{\mu \nu i k} \Sigma_{\gamma} U_{\alpha \gamma}\left(\phi_{i}, \theta_{i}\right) U_{\gamma \beta}^{+}\left(\phi_{k}, \theta_{k}\right)-\left(V_{\mu} \delta_{\alpha \beta}+I \sigma_{z \alpha \beta}\right) \delta_{\mu \nu} \delta_{i k}
$$

where the thermal spin disorder has been mapped onto random hopping integrals.

The low-temperature behavior of (1) is dominated by long-wavelength spin waves, where neighboring spins remain nearly parallel. Using $U\left(\varphi_{k}, \theta_{k}\right) \approx U\left(\varphi_{i}, \theta_{i}\right)$ and exploiting the identity $\mathrm{U}\left(\varphi_{i}, \theta_{i}\right) \mathrm{U}^{+}\left(\varphi_{i}, \theta_{i}\right)=\mathrm{I}$, we find that $\mathrm{U}\left(\varphi_{i}, \theta_{i}\right) \mathrm{U}^{+}\left(\varphi_{k}, \theta_{k}\right) \approx \mathrm{I}$. This means that the transformed hopping integrals are very similar to those at zero temperature, and the total wavefunction obtained via $\bigcup_{t}$ is a good approximation. Figure 1(b) shows a typical density of states for nonzero spin disorder. Compared to figure 1(a), the $\downarrow$ DOS exhibits minor distortions, but in the $\uparrow$ gap the DOS changes qualitatively, from zero to nonzero. An estimate for the magnitude of this spin-mixing contribution to the DOS is

$$
D_{\uparrow}(E) \approx \frac{M(0)-M(T)}{M(0)+M(T)} D_{\downarrow}(E)
$$


where $M(T)$ is the magnetization. This means that the resistance of the $\uparrow$ channel changes from infinity to a finite value.

It is important to note that equations (2) and (4) include intersublattice interactions, global noncollinearities, and hybridization effects on a tight-binding level. This can be seen from the hopping integrals $t_{\mu v i k}$, which depend on both the involved atomic orbitals ( $\mu$ and $v)$ and on the sublattice affiliation of the neighboring atoms $(i$ and $k$ ). However, the hopping integrals determine whether the system is ferromagnetic or paramagnetic, and in the derivation of (5) it is assumed that the atomic moments rotate without change in magnitude (strong ferromagnetism). This is reasonable for low temperatures but breaks down when finite-temperature excitations start to distort the density of states [3].

\section{Sublattice effects}

A striking feature of most or all half metals is the strong temperature dependence of the spin polarization, and (5) contributes to this dependence. One reason is that the temperature dependence of the spin polarization of half-metallic ferromagnets is more pronounced than in ordinary metallic ferromagnets. In this section, we consider half-metallic ferromagnets that contain two or more magnetic sublattices with temperature-independent atomic moments, whereas section 5 focuses on mechanisms that may reduce the magnetic moment.

Half metals have relatively complicated crystal structures, such as Heusler $\left(\mathrm{Mn}_{2} \mathrm{VAl}\right)$, semi-Heusler ( $\mathrm{NiMnSb})$, and rutile $\left(\mathrm{CrO}_{2}\right)$ structures, and their magnetism cannot be reduced to that of simple metallic magnets [16]. In particular, atoms belonging to nonequivalent crystallographic or magnetic sites have different magnetic moments (sublattice formation). An extreme but rather trivial case is bulk antiferromagnetism, where the $\uparrow$ and $\downarrow$ spin channels are equivalent and nothing is gained by using spin-polarized electrons. Cases of interest in half-metallic ferromagnetism are intermediate between ferromagnetism and antiferromagnetism and include both collinear ferromagnets or ferrimagnets and noncollinear spin structures. There are three reasons for the importance of these structures. First, from other ferromagnets it is known that sublattices have a strong effect on the temperature dependence of the net magnetization $[8,26]$. In small magnetic fields, such as of interest in the present context, this effect may be very pronounced, but high magnetic fields tend to align sublattices and smooth the corresponding noncollinearities. This enhances the magnetization well below $T_{\mathrm{c}}$. Figure 4 illustrates this point by comparing $M(T)$ curves for zero magnetic field (solid line) and moderate to high magnetic fields (dashed line). Second, noncollinearities are harmful to spin-dependent transport, because they yield a second spin channel for conduction. Third, different transition-metal sublattices yield, in general, different spin-scattering contributions per atom.

There are generally many sublattices. On a mean-field level, a system with $N$ sublattices yields $N$ nonlinear equations with $N$ unknowns [27], and the determination of the $\mathrm{Cu}$ rie temperature amounts to the diagonalization of an $N \times N$ matrix $J_{i j}$, where $i$ and $j$ denote the sublattices. In ferromagnets (FMs), the corresponding eigenmodes are plane waves (spin waves), and the ground-state spin structure is characterized by the wavevector $k=0$. Figure 4 shows the temperature dependence of the magnetization for a typical two-sublattice ferromagnet. In high field, the behavior is reminiscent of that of ordinary ferromagnets, but in low field, we see an inflection which strongly affects the $\downarrow$ DOS of equation (5).

In antiferromagnets (AFMs), $k=\pi / a$ describes an antiparallel spin orientation between nearest neighbors. As in some elemental rare earths, competing interactions may give rise to noncollinearities with incommensurate wavevectors, even in perfectly periodic crystals. Incommensurate means that the wavelength is unrelated to integer multiples of the lattice constant. For example, in NiMnSb, there is a magnon mode of wavevector $k_{o} a / 2 \pi \approx 0.3$, 




Figure 4. Magnetization of a two-sublattice magnet in high (dashed line) and low (solid line) fields. The noncollinearity behind the reduced low-field magnetization (solid line) reflects competing exchange (sections 4 and 5) rather than spin-orbit coupling (section 6).

corresponding to a magnetization angle of about $60^{\circ}$ between next-nearest $\mathrm{Mn}$ neighbors $[3,4]$. Note that this noncollinearity reflects to competing exchange and must not be confused with the noncollinearity due to Dzyaloshinskii-Moriya interactions [16].

Commensurate and incommensurate magnetization modes in half metals (section 6) are important, because they yield spin mixing. In $k$-space, the whole spectrum of $k$-values is necessary to explain the spin structure of the system. A semiquantitative two-level solution is to restrict the consideration to the most important wavevectors: $\mathbf{k}=0$ and $\mathbf{k}=\mathbf{k}_{o}$. Projecting the problem onto the two modes yields the equations [16]

$$
\langle m\rangle=\frac{\langle f\rangle}{h+\Delta J / 2}
$$

and

$$
m_{o}=\frac{f_{o}}{h-\Delta J / 2} .
$$

Here $\langle m\rangle$ and $\langle f\rangle$ are the transverse-magnetization and random-thermal-force volume averages, whereas $m_{o}$ and $f_{o} \sim \int \exp \left(\mathrm{ik}_{o} \cdot \mathbf{r}\right) f(\mathbf{r}) \mathrm{d} V$ are the amplitude of the noncollinear mode and the corresponding Fourier-transformed inhomogeneity, respectively, and the (positive) eigenvalue difference $J$ describes the relative stability of the noncollinear state.

Equation (6) shows that the noncollinear exchange $(\Delta J)$ suppresses ferromagnetic excitations (a) but enhances and stabilizes the noncollinear mode (b). In a very large positive field, both $\langle\mathbf{m}\rangle$ and $\mathbf{m}_{o}$ are zero, that is, $M_{z}$ is close to saturation. With reduced field strength $h$, both the volume-averaged random forces, $\langle\mathbf{f}\rangle$, and the random-force projections onto the chiral mode, $\mathbf{f}_{o}$, tend to destabilize the ferromagnetic state. However, due to the opposite sign of the $\Delta J$ contribution, the incommensurate mode is more enhanced; when $h=D J / 2$, then the saturated or aligned state becomes unstable, and the magnet becomes a "spin colloid" [16]. Such noncollinear modes may also be of interest in other areas of technology, such as room-temperature magnetic cooling, because they are able to absorb a certain amount of heat (magnetic entropy). 


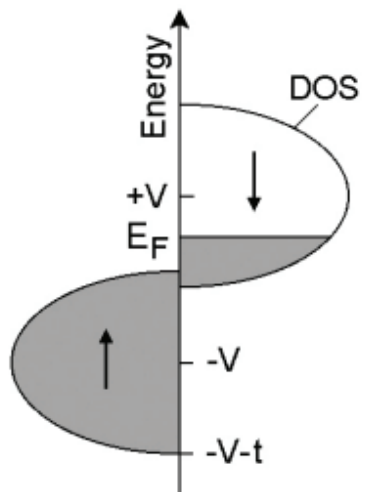

(a)

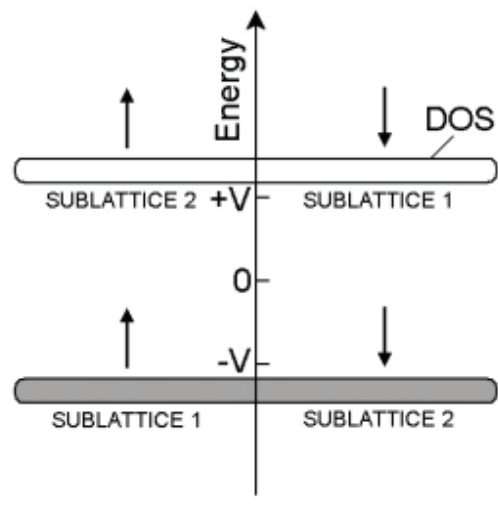

(b)

Figure 5. Simplified DOSs for (a) ferromagnetic and (b) antiferromagnetic order. This example illustrates how the DOSs depend on the local magnetization direction.

\section{Band-structure and correlation effects}

Sections 3 and 4 have focused on thermal excitations where the magnitude of the atomic moment is conserved, assuming that the localized regime of section 3 is more adequate than the opposite itinerant regime outlined in figure 2 . The localized regime is a fair starting point for many magnets, but in reality there is always some moment reduction. First, in a one-electron picture, any attempt to change the angle between the moments of neighboring atoms may reduce the moment. The VWI ferromagnetism discussed in section 2 is a drastic case, but similar phenomena occur in half-metallic ferromagnets. Second, solidstate magnetism is caused by electron-electron interactions, but one-electron calculations ignore correlation corrections and may overestimate the magnetic moment. For example, figures 1(a) and (c) illustrate how nonquasiparticle excitations [12, 15] change the density of states (DOS) near the Fermi level. This section deals with the relation between bandstructure effects and electron- electron interactions (correlations). By analyzing one-electron effects (section 5.1), non-Fermiliquid contributions (section 5.2), and Kondo-type interactions (section 5.3) it is argued that correlation effects are relatively unimportant in typical half-metallic magnets.

\subsection{Band structure and magnetic order}

Spin inhomogeneities may alter the DOS beyond the Stoner shift shown in figure 2. An instructive example is the extreme limit of antiferromagnetic order, where nearest-neighbor spins belong to different sublattices (1 and 2) of antiparallel spin orientation. This effect is actually encountered in manganites, where ferromagnetic (FM) and antiferromagnetic (AFM) configurations compete [9]. Figure 5 compares the schematic DOS for the FM (a) and AFM (b) cases. The origin of the big difference is well understood. In antiferromagnets, each second spin has the "wrong" orientation, because the Stoner or $V$ term in equation (2) changes sign. Hopping onto neighboring sites is therefore unfavorable, and the AFM bands are much narrower than the FM bands.

The difference between the FM and AFM DOSs in figure 5 is the main reason for the frequent occurrence of antiferromagnetism in the middle of the $3 \mathrm{~d}$ series, as exemplified by elemental Mn. It also explains why FM and AFM manganites are conducting and insulating, respectively, and is one aspect of the double exchange [28, 29] in manganites (section 5.3). 


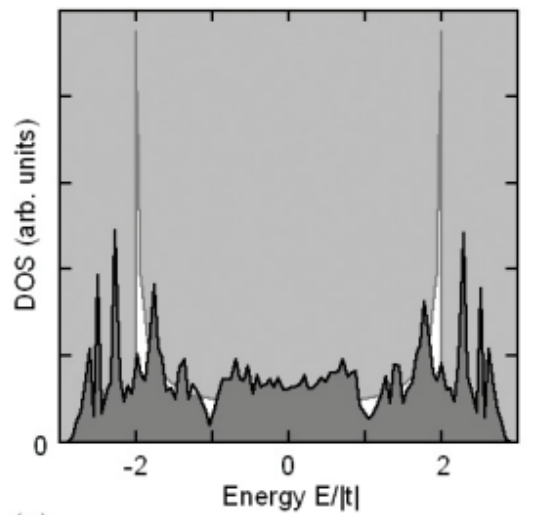

(a)

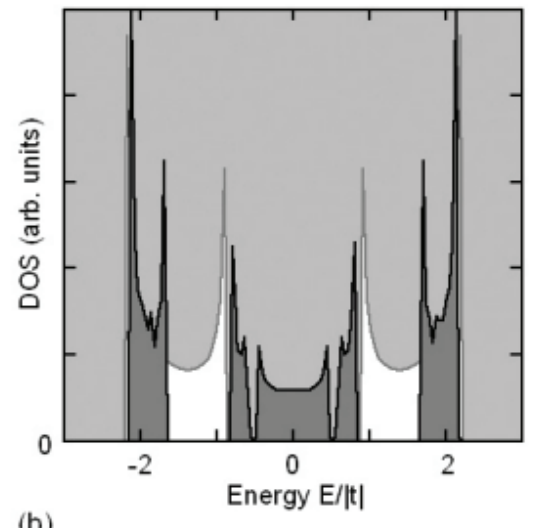

(b)

Figure 6. Densities of states in a one-dimensional spin model. (a) Ferromagnet (bright) and Curie paramagnet (dark), and (b) antiferromagnet (bright) and incommensurate magnet with $k a / 2 \pi=0.3$ (dark). In all cases, only one spin direction is shown.

To gauge the effect of thermally disordered and incommensurate spin states on the band structure, we exploit the random-alloy analogy of spin disorder, where A and B atoms correspond to $\uparrow$ and $\downarrow$ moments. The effect is seen most easily in one dimension: the effect of disorder increases with decreasing dimensionality. Figure 6 compares the DOSs for various configurations. Only one spin direction is shown, and the ratio $V / t$ of on-site exchange to hopping is equal to 0.9. The swallowtail shape of the ferromagnetic DOS is a one-dimensional band-structure effect, but otherwise the bright (FM and AFM) regions in figure 6 are equivalent to figure 5. The peaks and valleys in the dark regions of figure 6 reflect electronic Bragg reflections (a) at well-aligned spins $\left(\theta \approx 0^{\circ}\right.$ or $\left.180^{\circ}\right)$ and (b) at the noncollinear spin distribution. The dark regions exhibit a particularly strong dependence on the details of the model and, in (b), on the wavevector of the magnons.

In spite of the drastic band-structure changes, the atoms may keep their magnetic moments. The random-alloy or "paramagnetic" DOS in figure 6 is similar to the modification of the band structure of rare-earth magnets due to $\mathrm{Gd}^{3+}$ ions [30], although the metallic $\mathrm{Gd}^{3+}$ moment is always conserved, whereas $3 \mathrm{~d}$ moments may or may not be conserved. For example, first-principles calculations indicate that Mn moments in the half-metallic alloy NiMnSb are very stable, whereas the Ni moment easily collapses [17]. The Ni moment $\left(0.26 \mu_{\mathrm{B}}\right)$ is much smaller than the Mn moment $\left(3.7 \mu_{\mathrm{B}}\right)$ and contributes very little to the magnetization, but the Ni plays an important role in the formation of the hybridization gap. This is an example of a thermally distorted band structure mentioned at the end of section 3 . However, the assumption of pronounced spin misalignment, epitomized by figure 1 in [17], implies a contribution similar to figures 1(b) and 4, and blurs the distinction between spin mixing and band-structure effects.

A simpler and physically more transparent interpretation is that the thermally reduced Mn magnetization yields a diminished Mn exchange splitting. Straightforward bookkeeping for weak ferromagnets with Stoner-type rectangular bands of width $W$ then yields an approximate relation for the temperature dependence of the hybridization gap,

$$
\Delta(T)=\Delta(0)-\frac{W}{5}(m(0)-m(T))
$$

where $m(T)$ is the Mn magnetization measured in $\mu_{\mathrm{B}}$ per atom. The gap reaches zero at a transition temperature $T^{*}$ obtained from $m\left(T^{*}\right)=m(0)-5 \Delta(0) / W$. For typical half-metal- 
lic ferromagnets, $5 \Delta(0) / W<1$, and the gap closes well below the Curie temperature. The effect is most pronounced for narrow gaps and wide bands, as contrasted to the regime considered in section 3 , where the band width and gap are comparable. A very similar mechanism exists in strong ferromagnets, which leads to the conjecture that thermal gap closure is a typical rather than exceptional new feature.

Incidentally the behavior of NiMnSb is very similar to that encountered in $L 1_{0}$ alloys such as CoPt and FePt [31, 32], where the $3 \mathrm{~d}$ atoms spin polarize the $4 \mathrm{~d} / 5 \mathrm{~d}$ atoms and where the $4 \mathrm{~d} / 5 \mathrm{~d}$ moment collapses in an antiferromagnetic environment. In both cases, the unstable subsystem is an exchange-enhanced Pauli paramagnet, although nonlinear contributions are not necessarily negligible and series expansion [17] is a rather crude approach (see p 120 in [8]).

\subsection{Correlations and nonquasiparticle effects}

In the limit of strong correlations, there are stable magnetic moments (sections 3 and 4). The moments are often reproduced by one-electron calculations, which treat many-body interactions on a mean-field level (on-site Stoner exchange), but other properties, such as excitation energies and charge fluctuations, are less well described. More generally, strongly correlated magnets exhibit deviations from the Fermi-liquid or quasiparticle theory. A wellknown example is spin-charge separation [7,33], which means that the electron positions (charge degree of freedom) become largely independent of the spin direction.

Figure 1(c) shows that the nonquasiparticle states $[12,15]$ yield qualitatively new features. However, this does not necessarily mean that they yield strong deviations from (5). At low temperatures, the calculation by Irkhin et al. [12] reproduces the spin-mixing expression (5), but above a crossover temperature $T^{*}$, the magnetization is drastically reduced [12]. The crossover temperature scales as

$$
T^{*} \sim T_{\mathrm{c}} \frac{m^{2} \Delta_{\mathrm{s}}^{2}}{k_{\mathrm{F}}^{4}}
$$

When $I \sim \Delta_{\mathrm{S}}$ is much larger than $t \sim k_{\mathrm{F}}^{2} / m$, as assumed in the localized picture of sections 3 and 4, then $T^{*} \gg T_{\mathrm{c}}$ and the corrections due to (8) can be ignored. In the opposite limit, $T^{*}$ $\ll T_{\mathrm{c}}$, the finite-temperature magnetization undergoes a significant reduction.

Equation (8) indicates that the assumption of a well-conserved atomic spin splitting is not applicable for $T>T^{*}$ [12]. However, the reduction is a Stoner effect similar to (7) rather than a correlation effect, because $T^{*}$ decreases with $I$ and the magnetization reduction is most pronounced for small $I$, where standard band-structure and Fermi-liquid theories are applicable. Details of the DOS [12] affect the outcome of this band-structure modification but do not introduce correlations.

Note that Otto et al. [6] investigated the electrical resistivity and the Hall effect of various semi-Heusler alloys, including NiMnSb, and concluded that the local-band description is much more adequate than the itinerant or "weak-exchange" description. In their paper, the term local-band magnetism is defined very broadly, including the disordered local moment (DLM) picture [34] and anticipating the spin mixing of section 3.

\subsection{Kondo interactions}

An interesting correlation effect in systems containing both localized and extended electrons is the Kondo effect. In a narrow sense, the term refers to the resistance minimum observed in metals containing magnetic impurities, but the phenomenon also occurs in other correlated systems, such as heavy fermions and manganites. A key feature of the Kondo effect is the simultaneous involvement of strongly correlated electrons in localized orbitals 
$|l o c\rangle$ and weakly correlated electrons in extended orbitals $\mid$ ext $\rangle$ [7]. Examples of localized and extended orbitals, respectively, are the following pairs: (i) $3 \mathrm{~d}$ and $4 \mathrm{~s}$ electrons in dilute magnetic transition-metal alloys such as $\mathrm{Cu}_{1-x} \mathrm{Fe}_{x}$, (ii) $4 \mathrm{f}$ or $5 \mathrm{f}$ and conduction electrons in heavy-fermion compounds such as $\mathrm{CeAl}_{2}$, and (iii) $\mathrm{Mn}_{2 \mathrm{~g}}$ and $\mathrm{e}_{\mathrm{g}}$ and electrons in manganites. In the case of $\mathrm{Mn}$, the interaction gives rise to ferromagnetic double exchange, and a similar situation is encountered in the half-metallic oxide $\mathrm{CrO}_{2}$. The corresponding ferromagnetic spin alignment enhances not only the magnetization but also the conductivity (section 5.1). Since the Kondo effect involves strongly correlated electrons, it can be used to investigate correlations.

A comprehensive description of the Kondo effect requires the consideration of many electrons, but to see the essential many-body features of the phenomenon, it is sufficient to consider just two electrons in two orbitals [7]. It is convenient to start from the two-electron wavefunction

$$
\left.\left.\left.\left.\left.|\psi\rangle=c_{1} \mid \text { ext }\right\rangle \mid \text { ext }\right\rangle+c_{2} \mid \text { ext }\right\rangle|\operatorname{loc}\rangle+c_{3} \mid \text { loc }\right\rangle \mid \text { ext }\right\rangle+c_{4}|\operatorname{loc}\rangle|\operatorname{loc}\rangle .
$$

For example, the $c_{2}$-term means that the first and second electrons are in the extended and localized states, respectively. In terms of the basis defined by (9), the Hamiltonian is

$$
\mathcal{H}=\left(\begin{array}{cccc}
V & t & t & 0 \\
t & 0 & 0 & t \\
t & 0 & 0 & t \\
0 & t & t & U-V
\end{array}\right) .
$$

Here $V=E_{\text {ext }}-E_{\text {loc }}$ is the spin-independent orbital energy difference between the extended and localized electrons $(V>0), U \sim I$ is the Coulomb energy, and $t<0$ is the hopping integral between $\mid$ ext $\rangle$ and $|l o c\rangle$. The orbital energy $V$ favors the occupancy of the localized orbital, but the strong Coulomb repulsion $U>V$ makes the double occupancy of this orbital energetically unfavorable. Since $U$ is very large, we can ignore the admixture of $\mid$ loc $\rangle|\operatorname{loc}\rangle$ character to the system's low-lying states.

In the absence of hopping, the ground state of (10) is two-fold degenerate, corresponding to the wavefunctions $\left|\psi_{ \pm}\right\rangle=\mid$ext $\rangle|\operatorname{loc}\rangle \pm|\operatorname{loc}\rangle|\operatorname{ext}\rangle$. The function $\left|\psi_{+}\right\rangle$is symmetric and describes an antiferromagnetic (AFM) spin singlet $(\uparrow \downarrow, S=0)$, whereas the antisymmetric wavefunction $\left|\psi_{-}\right\rangle$corresponds to a ferromagnetic (FM) triplet $(\uparrow \uparrow, S=1)$. In the presence of hopping, the triplet remains unaffected $(E=0)$, but the AFM singlet, $\mid$ ext $\rangle|\operatorname{loc}\rangle+|\operatorname{loc}\rangle|\operatorname{ext}\rangle$, hybridizes with $\mid$ ext $\rangle \mid$ ext $\rangle$. This hybridization lowers the energy of the AFM state by $\left.2 t^{2}\right\rangle$ $V$ and means that the ground state is antiferromagnetic. Figure 7 shows the corresponding level splitting. Note that the FM state is unable to benefit from the hopping, because the Pauli principle excludes the double occupancy of any orbital by parallel spins.

In the ground state, the localized spin is compensated (screened) by the delocalized spin, but when the temperature exceeds the Kondo temperature $T_{\mathrm{K}}$, then the $\uparrow \downarrow$ and $\uparrow \uparrow$ configurations are both occupied and the net coupling between screening cloud and the localized spin vanish. Together with the accompanying resistance minimum near $T_{\mathrm{K}}$, this is a signature of the Kondo effect. Note that the Kondo temperature $2 t^{2} / V$ is formally independent of $U$ but is based on the assumption of strong correlations, $U=\infty$. Physically, the first and second electrons are attracted by the low orbital energy of the localized orbital (energy gain $V$ ), but the second electron is repelled by the strong Coulomb interaction of the first electron. In the one-electron approximation, the second electron does not know the position of the first electron, because it interacts with an average electron distribution. This overestimates the Coulomb energy.

Interestingly, the Kondo splitting of figure 7 is reproduced by an unrestricted HartreeFock (HF) approximation. The idea is to use a single Slater determinant constructed from arbitrary one-electron wavefunctions, as proposed by Coulson and Fischer [35]. In their 


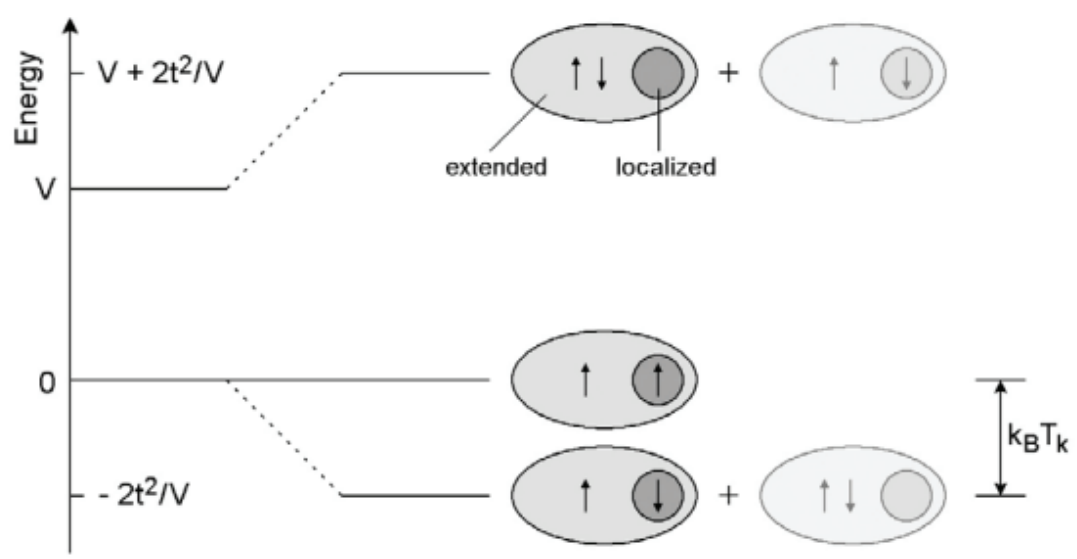

Figure 7. Kondo interactions in a half-metallic model. The bright gray configurations indicate admixture due to hybridization. Simplifying somewhat, the model is of type III, but similar effects exist in type-I half-metals, such as $\mathrm{CrO}_{2}$.

original form, the Fisher-Coulson wavefunctions describe a $\mathrm{H}_{2}$ molecule by partially localized one-electron orbitals, in contrast to "Bloch-symmetric" bonding and antibonding one-electron orbitals with symmetric electron densities. Describing the FM state by $\left|\psi_{1}\right\rangle=$ $\mid$ ext $\rangle$ and $\left|\psi_{2}\right\rangle=\mid$ loc $\rangle$, using the AFM ansatz $\left|\psi_{1}\right\rangle=\mid$ ext $\rangle$ and $\left|\psi_{2}\right\rangle=\mid$ loc $\rangle+c_{o} \mid$ ext $\rangle$, and minimizing the AFM energy yields $c_{o}=-E_{o} / 2 t$ and reproduces the Kondo splitting. Bloch-symmetric and unrestricted Hartree-Fock approximations are a notoriously unreliable tool to investigate excitations in correlated electron systems. However, in the present case, the relevant first excited state is ferromagnetic, and it is well-known that the Pauli principle takes care of correlations in the ferromagnetic state.

\section{Discussion and conclusions}

The experimental investigation of half metals poses a number of challenges, and a variety of questions remain to be answered. First, the detection of half-metallic ferromagnetism is more complicated than the detection of "simpler" phenomena, such as ordinary ferromagnetism and metallic conductivity [2]. Second, the phenomenon itself has been questioned on intrinsic and extrinsic grounds [3], and it seems that both real-structure imperfections and thermal disorder have a very negative effect on the spin polarization. Important real-structure or extrinsic features are atomic defects, grain boundaries, interfaces and nanoscale geometrical features, which all affect the local DOS. For example, spin mixing involving nanojunctions was investigated in [36].

A different question is the existence of intrinsic half-metallic ferromagnetism in perfect bulk materials. As pointed out in section 4, magnetic measurements are often performed in high magnetic fields; this overestimates the spin polarization by aligning the magnetic sublattices and makes the measurements less sensitive to a small spin minority density of states. The compound structure of half metals yields a number of low-energy transverse and longitudinal optical modes, which couple to spin-wave modes and reduce the net magnetization $[3,4]$. In real space, the magnetization modes correspond to a tilting between neighboring atomic spins, depending on the $k$-vector where the phonon and magnon bands cross. Generally, the closer to the Brillouin zone edge the magnons and transverse optical modes couple, the greater the tilt angle. The corresponding moment misalign- 
ment gives rise to a pronounced spin mixing (section 4). Examples of the involvement of phonons NiMnSb, $\mathrm{CrO}_{2}, \mathrm{Fe}_{3} \mathrm{O}_{4}$, and $\mathrm{La}_{0.7} \mathrm{Sr}_{0.3} \mathrm{MnO}_{3}$ [3, 4]. In the latter case, the spinwave softening is related to the proximity of the charge-ordered insulating state, that is, to the FM-AFM transition mentioned in section 5. The spin mixing is complemented by temperature-dependent changes in the density of states, both subband shifts, as in figure 2, and distortions of the shape, as in figure 6. Little is known about the relative depolarization contributions of the different mechanisms, and their investigation remains an ongoing challenge.

One aspect of this paper is the role of correlations. In the limit of strong intra-atomic exchange, the spin-mixing model of section 3 works well, although it fails to address specific correlation effects. Magnons are included and are well described in this strong-correlation regime, because changes in the density of the states caused by disorder and noncollinear spin states leave the atomic moments unchanged. In the opposite limit of relatively weak intra-atomic exchange, one-electron effects yield a pronounced modification of the atomic moments, similar to the moment reduction in very weak itinerant magnets. There are no magnons in the usual sense, especially at large wavevectors. If one tries to create a noncollinear state with large $k$-vector, then the magnetization collapses in this regime, rather than undergoing rotation. A good example is the collapse of the Ni moment discussed in section 5.1.

Half-metallic ferromagnets seem to exhibit a general trend towards magnetic instabilities, both locally and globally. This reflects the band structure of typical half-metals, with several nonequivalent sites or sublattices, competing ferromagnetic and antiferromagnetic interactions, and partially unstable atomic moments. Compared to alloys, moment reductions, or "high-spin-low-spin" transitions, may be less important in oxides, but oxides tend to exhibit relatively weak ferromagnetic or predominantly antiferromagnetic interatomic exchange [8]. In a crude approximation, the temperature dependence of the hybridization gap of both alloys and oxides is described by (7), so there are two ways of improving the spin polarization below $T_{\mathrm{c}}$ : (i) larger zero-temperature hybridization gaps and (ii) less pronounced interatomic hopping (narrow bands). Structural imperfections - such as defects and interfaces, short-wavelength thermal excitations, and phonons - amplify the effect of magnetic instabilities. Defects remain a nontrivial challenge in oxides, including $\mathrm{CrO}_{2}$, which is one of the few oxides with predominantly ferromagnetic exchange.

The spin structure is also influenced by spin-orbit coupling [8, 16, 27, 37, 38]. Spinorbit interactions are often small in simple ferromagnets but they become important when the leading exchange and hopping contributions nearly cancel each other. One example is Dzyaloshinskii-Moriya (DM) interactions [16, 27, 37], which occur not only in perfect crystals with broken inversion symmetry but also in spin glasses and nanostructures. Other examples are magnetocrystalline anisotropy and magnetostriction, which both originate from spin-orbit coupling and may enhance the noncollinearity of the spin state of polycrystalline and imperfect samples.

In conclusion, we have investigated the spin polarization of half-metallic ferromagnets. At finite temperatures, the picture is intermediate between the strongly correlated Heisenberg and weakly correlated one-electron limits. In addition to Heisenberg interactions with largely conserved magnetic moments and spin mixing, there are Stoner-type contributions, characterized by reduced atomic moments, and complicated distortions of the DOS. In the simplest model, the Stoner mechanism means that the hybridization gap closes due to reduced exchange splitting between majority and minority bands. These mechanisms all contribute to the widespread thermal depolarization and are enhanced by the presence of two or more sublattices in typical half metals. Correlations are analyzed by a version of the Kondo model and by an unrestricted Hartree-Fock approximation, which becomes exact for the considered Kondo problem. However, correlation effects are relatively unimportant 
in typical half-metals, and the essential physics of finite-temperature depolarization is contained in the one-electron picture.

\section{Acknowledgments}

This work has been supported by NSF-MRSEC, NCNM and, partially, NCESR. The author is grateful to P. A. Dowben for stimulating discussions.

\section{References}

[1] Groot R. D. and Buschow K. H. J. 1986 J. Magn. Magn. Mater. 54-57 1377

[2] Coey J. M. D. and Venkatesan M. J. 2002 J. Appl. Phys. 918345

[3] Dowben P. A. and Skomski R. 2004 J. Appl. Phys. 957453

[4] Borca C. N, Komesu T., Jeong H-K., Dowben P. A., Ristoiu D., Hordequin Ch., Pierre J., and Nozières J. P. 2000 Appl. Phys. Lett. 7788

[5] Watts S. M., Wirth S., von Molnár S., Barry A., and Coey J. M. D. 2000 Phys. Rev. B 619621

[6] Otto M. J., Feil H., van Woerden R. A. M., Wijngaard J., van der Valk P., van Bruggen C., and Haas C. J. 1987 J. Magn. Magn. Mater. $\mathbf{7 0} 33$

[7] Fulde P. 1991 Electron Correlations in Molecules and Solids (Berlin: Springer)

[8] Skomski R. and Coey J. M. D. 1999 Permanent Magnetism (Bristol: Institute of Physics Publishing)

[9] Coey J. M. D., Viret M., and von Molnár S. 1999 Adv. Phys. 48167

[10] Ziese M. and Thornton M. J. (ed.) 2001 Spin Electronics (Berlin: Springer)

[11] Donath M. and Nolting W. (eds.) 2005 Local-Moment Ferromagnets (Berlin: Springer)

[12] Irkhin V. Yu., Katsnelson M. I., and Lichtenstein A. I. 2005 Nonquasiparticle states in half-metallic ferromagnets Local-Moment Ferromagnets ed. M. Donath and W. Nolting (Berlin: Springer) pp 217-43

[13] Skomski R. and Dowben P. A. 2002 Europhys. Lett. 58544

[14] Dowben P. A. and Skomski R. 2003 J. Appl. Phys. 937948

[15] Chioncel L., Katsnelson M. I., de Groot R. A., and Lichtenstein A. I. 2003 Phys. Rev. B 68 144425-1

[16] Skomski R, Zhou J., Dowben P. A., and Sellmyer D. J. 2005 J. Appl. Phys. 97 10C305-1

[17] Ležaić M., Mavropoulos Ph., Enkovaara J., Bihlmayer G., and Blügel S. 2006 Phys. Rev. Lett. 97026404

[18] Skomski R., Komesu T., Borca C. N., Jeong H-K., Dowben P. A., Ristoiu D., and Nozières J. P. 2001 J. Appl. Phys. 897275

[19] Yu P. Y. and Cardona M. 1999 Fundamentals of Semiconductors (Berlin: Springer)

[20] Gubanov V. A., Liechtenstein A. I., and Postnikov A. V. 1992 Magnetism and the Electronic Structure of Crystals (Berlin: Springer)

[21] Murata K. K. and Doniach S. 1972 Phys. Rev. Lett. 29285

[22] Moriya T. 1985 Spin Fluctuations in Itinerant Electron Magnetism (Berlin: Springer)

[23] Györffy B. L, Pindor A. J., Staunton J., Stocks G. M., and Winter H. 1985 J. Phys. F: Met. Phys. 151337

[24] Qi Q-N., Skomski R., and Coey J. M. D. 1994 J. Phys.: Condens. Matter 63245

[25] Mohn P. 2003 Magnetism in the Solid State (Berlin: Springer)

[26] Smart J. S. 1966 Effective Field Theories of Magnetism (Philadelphia, PA: Saunders)

[27] Skomski R. 2003 J. Phys.: Condens. Matter 15 R841

[28] Anderson P. W. and Hasegawa H. 1955 Phys. Rev. 100675

[29] de Gennes P-G. 1960 Phys. Rev. 118141

[30] Sandratski L. M. and Kübler J. 1998 Magnetism and Electronic Correlations in Local-Moment Systems: Rare-Earth Elements and Compounds ed. M. Donath, P. A. Dowben, and W. Nolting (Singapore: World Scientific) pp 271-91

[31] Skomski R., Kashyap A., and Sellmyer D. J. 2003 IEEE Trans. Magn. 392917

[32] Mryasov O. N., Nowak U., Guslienko K. Y., and Chantrell R. 2005 Europhys. Lett. 69805

[33] Schofield A. J. 1999 Contemp. Phys. 4095

[34] Hubbard J. 1979 Phys. Rev. B 19 2626; Hubbard J. 1979 Phys. Rev. B 204584

[35] Coulson C. A. and Fisher I. 1949 Phil. Mag. 40386

[36] MacDonald A. H., Jungwirth T., and Kasner M. 1998 Phys. Rev. Lett. 81705

[37] Fischer K-H. and Hertz A. J. 1991 Spin Glasses (Cambridge: Cambridge University Press)

[38] Sandratski L. M. 2001 Phys. Rev. B 64134402 